\title{
MELTING OF THE EUCLIDEAN METRIC TO NEGATIVE SCALAR CURVATURE
}

\author{
JoNGSU KIM
}

\begin{abstract}
We find a $C^{\infty}$-continuous path of Riemannian metrics $g_{t}$ on $\mathbb{R}^{k}, k \geq 3$, for $0 \leq t \leq \varepsilon$ for some number $\varepsilon>0$ with the following property: $g_{0}$ is the Euclidean metric on $\mathbb{R}^{k}$, the scalar curvatures of $g_{t}$ are strictly decreasing in $t$ in the open unit ball and $g_{t}$ is isometric to the Euclidean metric in the complement of the ball. Furthermore we extend the discussion to the Fubini-Study metric in a similar way.
\end{abstract}

\section{Introduction}

In a remarkable paper [11], Lohkamp has made the following conjecture in Riemannian geometry.

Conjecture. Let $\left(M^{k}, g_{0}\right), k \geq 3$, be a manifold and $B \subset M$ a ball. Then there is a $C^{\infty}$-continuous path of Riemannian metrics $g_{t}, 0 \leq t \leq \varepsilon$, on $M$ with

(i) Ricci curvature of $g_{t}$ is strictly decreasing in $t$ on $B$.

(ii) $g_{t} \equiv g_{0}$ on $M \backslash B$.

If such a path $g_{t}$ exists, we call it a Ricci-curvature melting of $g_{0}$ on $B$. This conjecture, if true, would certainly imply a scalar-curvature melting, meaning a path $g_{t}$ as above but with scalar curvature replacing the Ricci curvature in the condition (i). We note that common metric-surgery arguments do not seem to yield a scalar-curvature melting. If one considers the scalar curvatures $s\left(g_{t}\right)$ for a scalar-curvature melting $g_{t}$, then $\left.\frac{d s\left(g_{t}\right)}{d t}\right|_{t=0} \leq 0$ on $B$. In this way, the scalar-curvature melting is related to the deformation theory of the scalar curvature functional [4, Chapter 4]. A remarkable approach is the theory of local scalar curvature deformation of J. Corvino [6, Theorem 4]. He considered the formal adjoint $L_{g}^{*}$ of the linearization $L_{g}$ of the scalar curvature functional on the space of Riemannian metrics restricted to a domain. According to his work, a scalar-curvature melting of $g$ seems to exist when $L_{g}^{*}$ is injective. Years

Received March 12, 2012; Revised July 18, 2012.

2010 Mathematics Subject Classification. 53B20, 53C20, 53C21.

Key words and phrases. scalar curvature.

This research was supported by Basic Science Research Program through the National Research Foundation of Korea(NRF) funded by the Ministry of Education, Science and Technology(2011-0005248). 
later, this injectivity condition of $L_{g}^{*}$ on domains was shown to be a generic one by Beig, Chruściel and Schoen (see Theorem 6.1 and Theorem 7.4 in [3]). Now the question is how to melt a Riemannian metric which does not satisfy this condition.

In this context, Euclidean metrics arise importantly because they are outstanding ones, not satisfying this condition. In a recent paper [8], we explained the scalar-curvature melting of Euclidean metric in 3 dimension. The purpose of this article is to complete the scalar-curvature melting of Euclidean metrics in any dimension $\geq 3$ and then extend the discussion to the Fubini-Study metric in a similar way.

We shall first construct a family of Riemannian metrics on $\mathbb{R}^{k}, k \geq 3$ which have negative scalar curvatures on a pre-compact (open) set and are Euclidean away from it. In even dimension we already have such a family of metrics [7]. In odd dimension, we use the coordinates $\left(r_{1}, \theta_{1}, \ldots, r_{n}, \theta_{n}, z\right)$ on $\mathbb{R}^{2 n+1}$ where $\left(r_{i}, \theta_{i}\right)$ are the polar coordinates on the $i$-th direct summand of $\mathbb{R}^{2 n+1}:=$ $\mathbb{R}^{2} \times \cdots \times \mathbb{R}^{2} \times \mathbb{R}$ and $z$ is the coordinate for the last summand $\mathbb{R}$. We express the Euclidean metric as $g_{0}=\sum_{i=1}^{n}\left(d r_{i}^{2}+r_{i}^{2} d \theta_{i}^{2}\right)+d z^{2}$. We deform it to $g=\sum_{i=1}^{n}\left(f_{i}^{2} d r_{i}^{2}+\frac{r_{i}^{2}}{f_{i}^{2}} d \theta_{i}^{2}\right)+d z^{2}$ and choose smooth functions $f_{i}$ so that $g$ has negative scalar curvature on a pre-compact set near origin and is Euclidean away from it.

Then by conformal change of $g$ (also for the even dimensional metrics mentioned above), we spread the negativity inside the pre-compact set over to a larger ball. In the process, we found a natural choice of parameter $t$ to get $g_{t}$. In this way we get a scalar-curvature melting:

Theorem 1.1. There exists a $C^{\infty}$-continuous path of Riemannian metrics $g_{t}$ on $\mathbb{R}^{k}, k \geq 3$ which exists for $0 \leq t \leq \varepsilon$ for some number $\varepsilon$ with the following property: $g_{0}$ is the Euclidean metric on $\mathbb{R}^{k}, s\left(g_{\tilde{t}}\right)<s\left(g_{t}\right)$ for $0 \leq t<\tilde{t} \leq \varepsilon$ in the open unit ball and $g_{t}$ is the Euclidean metric in the complement of the ball.

In Section 2, we construct Riemannian metrics on $\mathbb{R}^{2 n+1}$ that have negative scalar curvatures on a pre-compact set and are Euclidean away from it. In Section 3 , we demonstrate a $C^{\infty}$-continuous path of metrics $g_{t}$ such that the scalar curvature $s\left(g_{t}\right)$ is monotonically decreasing in $t$. In Section 4 , by a conformal deformation we get a genuine scalar-curvature melting on the unit ball in $\mathbb{R}^{2 n+1}$. We also observe that similar argument works for even dimensions. In Section 5 we discuss the Fubini-Study metric in a similar way.

\section{Construction of the metric}

We will deform the Euclidean metric $g_{0}=\sum_{i=1}^{n}\left(d r_{i}^{2}+r_{i}^{2} d \theta_{i}^{2}\right)+d z^{2}$ on $\mathbb{R}^{2 n+1}=\mathbb{R}^{2} \times \cdots \times \mathbb{R}^{2} \times \mathbb{R}$ to a metric of the form

$$
\tilde{g}=\sum_{i=1}^{n}\left(f_{i}^{2} d r_{i}^{2}+\frac{r_{i}^{2}}{f_{i}^{2}} d \theta_{i}^{2}\right)+d z^{2},
$$


where $f_{i}$ 's are smooth positive functions on $\mathbb{R}^{2 n+1}$ depending only on the variables $r_{1}, \ldots, r_{n}, z . \quad \tilde{g}$ is a metric on $\mathbb{R}^{2 n+1} \backslash\left\{\left(r_{1}, \theta_{1}, \ldots, r_{n}, \theta_{n}, z\right) \mid r_{i}=\right.$ 0 for some $i$ \}. Below we shall choose $f_{i}$ so that $\tilde{g}$ is smooth on $\mathbb{R}^{2 n+1}$. Let $e_{2 i-1}=\frac{1}{f_{i}} \frac{\partial}{\partial r_{i}}, e_{2 i}=\frac{f_{i}}{r_{i}} \frac{\partial}{\partial \theta_{i}}, i=1,2, \ldots, n, e_{2 n+1}=\frac{\partial}{\partial z}$.

Let $\omega_{i}$ be the dual co-frame fields of $e_{i}: \omega_{2 i-1}=f_{i} d r_{i}, \omega_{2 i}=\frac{r_{i}}{f_{i}} d \theta_{i}$, $\omega_{2 n+1}=d z$. We compute the connection 1-forms $\omega_{i j}$ with respect to $\omega_{i}$ : $d \omega_{i}=\sum_{j=1}^{2 n+1} \omega_{i j} \wedge \omega_{j}$, with $\omega_{i j}=-\omega_{j i}$; one may compute

$$
2 a_{i j k}=\left\langle d \omega_{k}, \omega_{i} \wedge \omega_{j}\right\rangle_{g}-\left\langle d \omega_{i}, \omega_{j} \wedge \omega_{k}\right\rangle_{g}-\left\langle d \omega_{j}, \omega_{k} \wedge \omega_{i}\right\rangle_{g},
$$

where $\omega_{i j}=\sum_{k=1} a_{i j k} \omega_{k}$. We get

$$
\begin{aligned}
d \omega_{2 n+1} & =0, \\
d \omega_{2 i-1} & =\frac{f_{i, 2 n+1}}{f_{i}} \omega_{2 n+1} \wedge \omega_{2 i-1}+\sum_{j=1}^{n} \frac{f_{i, j}}{f_{i} f_{j}} \omega_{2 j-1} \wedge \omega_{2 i-1} \text { and } \\
d \omega_{2 i} & =-\frac{f_{i, 2 n+1}}{f_{i}} \omega_{2 n+1} \wedge \omega_{2 i}+\sum_{j=1}^{n} \frac{\delta_{i j} f_{i}-r_{i} f_{i, j}}{r_{i} f_{i} f_{j}} \omega_{2 j-1} \wedge \omega_{2 i} \\
\text { for } i & =1,2, \ldots, n .
\end{aligned}
$$

Here we write $f_{i, j}=\frac{\partial f_{i}}{\partial r_{j}}, \quad f_{i, j k}=\frac{\partial^{2} f_{i}}{\partial r_{k} \partial r_{j}}$. Then we can get $\omega_{2 i-12 j-1}=$ $-\frac{f_{i, j}}{f_{j} f_{i}} \omega_{2 i-1}+\frac{f_{j, i}}{f_{j} f_{i}} \omega_{2 j-1}, \omega_{2 i-1} 2 j=\frac{\delta_{j i} f_{j}-r_{j} f_{j, i}}{r_{j} f_{i} f_{j}} \omega_{2 j}$ and $\omega_{2 i} 2 j=0$ for $i, j=$ $1,2, \ldots, n$ and $\omega_{2 n+12 i-1}=\frac{f_{i, 2 n+1}}{f_{i}} \omega_{2 i-1}, \omega_{2 n+1} 2 i=-\frac{f_{i, 2 n+1}}{f_{i}} \omega_{2 i}$. We use the formula $d \omega_{i j}-\omega_{i k} \wedge \omega_{k j}=\sum_{k<l}^{2 n+1} R_{i j k l} \omega_{k} \wedge \omega_{l}$ to compute the curvature components;

$$
\begin{aligned}
& R_{2 i-12 j-1} 2 j-12 i-1 \\
= & \left(-d \omega_{2 i-1} 2 j-1+\omega_{2 i-1} \wedge \omega_{s} 2 j-1, \omega_{2 i-1} \wedge \omega_{2 j-1}\right)_{g} \\
= & -\frac{f_{i, j j}}{f_{i} f_{j}^{2}}+\frac{f_{i, j} f_{j, j}}{f_{i} f_{j}^{3}}-\frac{f_{j, i i}}{f_{j} f_{i}^{2}}+\frac{f_{j, i} f_{i, i}}{f_{j} f_{i}^{3}}-\sum_{k \neq i, j}^{n} \frac{f_{i, k} f_{j, k}}{f_{i} f_{j} f_{k}^{2}}-\frac{f_{i, 2 n+1}}{f_{i}} \cdot \frac{f_{j, 2 n+1}}{f_{j}}, \\
& R_{2 i 2 j 2 j} 2 i \\
= & \left(-d \omega_{2 i} 2 j, \omega_{2 i} \wedge \omega_{2 j}\right)_{g}+\left(\omega_{2 i} \wedge \wedge \omega_{s} 2 j, \omega_{2 i} \wedge \omega_{2 j}\right)_{g} \\
= & \frac{f_{j, i}}{r_{i} f_{i}^{2} f_{j}}+\frac{f_{i, j}}{r_{j} f_{j}^{2} f_{i}}-\sum_{k=1}^{n} \frac{f_{i, k} f_{j, k}}{f_{i} f_{j} f_{k}^{2}}-\frac{f_{i, 2 n+1}}{f_{i}} \cdot \frac{f_{j, 2 n+1}}{f_{j}}, \\
& R_{2 i-1} 2 j 2 j 2 i-1 \\
= & \left(-d \omega_{2 i-1} 2 j, \omega_{2 i-1} \wedge \omega_{2 j}\right)_{g}+\left(\omega_{2 i-1} \wedge \omega_{s} 2 j, \omega_{2 i-1} \wedge \omega_{2 j}\right)_{g} \\
= & \frac{\delta_{i j} f_{i, i}}{r_{j} f_{i}^{3}}+\frac{f_{j, i i}}{f_{i}^{2} f_{j}}-\frac{f_{j, i} f_{i, i}}{f_{i}^{3} f_{j}}-\frac{2 f_{j, i}^{2}}{f_{i}^{2} f_{j}^{2}}+\frac{2 \delta_{i j} f_{j, i}}{r_{j} f_{i}^{2} f_{j}}-\sum_{k \neq i}^{n} \frac{\delta_{j k} f_{i, k}}{r_{j} f_{i} f_{k}^{2}}+\sum_{k \neq i}^{n} \frac{f_{i, k} f_{j, k}}{f_{i} f_{j} f_{k}^{2}} \\
& +\frac{f_{i, 2 n+1}}{f_{i}} \cdot \frac{f_{j, 2 n+1}}{f_{j}},
\end{aligned}
$$




$$
\begin{aligned}
& R_{2 n+12 i-12 i-12 n+1}=-\left(\frac{f_{i, 2 n+1}}{f_{i}}\right)_{2 n+1}-\left(\frac{f_{i, 2 n+1}}{f_{i}}\right)^{2}, \\
& R_{2 n+12 i 2 i 2 n+1}=\left(\frac{f_{i, 2 n+1}}{f_{i}}\right)_{2 n+1}-\left(\frac{f_{i, 2 n+1}}{f_{i}}\right)^{2} .
\end{aligned}
$$

The scalar curvature is as follows;

$$
\begin{aligned}
\frac{s_{\tilde{g}}}{2}= & \sum_{1 \leq s<t}^{2 n+1} R_{s t t s} \\
= & \sum_{1 \leq t}^{2 n} R_{2 n+1} t t 2 n+1+\sum_{1 \leq i<j}^{n}\left(R_{2 i-1} 2 j-12 j-12 i-1+R_{2 i} 2 j 2 j 2 i\right) \\
& +\sum_{1 \leq i<j}^{n}\left(R_{2 i-1} 2 j 2 j 2 i-1+R_{2 j-1} 2 i 2 i 2 j-1\right)+\sum_{i=1}^{n} R_{2 i-1} 2 i 2 i 2 i-1 \\
= & -\sum_{i=1}^{n}\left(\frac{f_{i, 2 n+1}}{f_{i}}\right)^{2}+\sum_{i=1}^{n}\left(\frac{f_{i, i i}}{f_{i}^{3}}+3 \frac{f_{i, i}}{r_{i} f_{i}^{3}}-3 \frac{f_{i, i}^{2}}{f_{i}^{4}}\right)-\sum_{i<j} \frac{f_{i, j}^{2}+f_{j, i}^{2}}{f_{i}^{2} f_{j}^{2}} \\
= & -\frac{1}{2} \sum_{i=1}^{n}\left\{\left(f_{i}^{-2}\right)_{i i}+\frac{3}{r_{i}}\left(f_{i}^{-2}\right)_{i}\right\}-\sum_{i<j} \frac{f_{i, j}^{2}+f_{j, i}^{2}}{f_{i}^{2} f_{j}^{2}}-\sum_{i=1}^{n}\left(\frac{f_{i, 2 n+1}}{f_{i}}\right)^{2} .
\end{aligned}
$$

Set $F_{i}=f_{i}^{-2}, i=1, \ldots, n$. We shall find the functions $F_{i}$ so that they satisfy

$$
\sum_{i=1}^{n}\left(F_{i, i i}+\frac{3}{r_{i}} F_{i, i}\right)=0 .
$$

We consider smooth functions $\beta(z)$ and $\alpha_{j}^{i}(r), i=1, \ldots, n-1, j=1, \ldots, n$ on $\mathbb{R}$ which satisfy at least

$$
\begin{gathered}
\beta(z)=0 \text { for } z \leq-1, \text { or } z \geq 1, \quad \text { and } \quad \beta(z)>0 \text { on }-1<z<1, \\
\alpha_{j}^{i}(r)=0 \text { for } r \leq 0, \text { or } r \geq 1 .
\end{gathered}
$$

The functions $\alpha_{j}^{i}$ 's need to be specified more. Let $k_{j}^{i}(r)$ be smooth functions on $\mathbb{R}$ satisfying

$$
\left\{\begin{array}{l}
\text { a) } k_{j}^{i}(r)=0 \text { for } r \leq 0, r \geq 1, \\
\text { b) }\left|\left(k_{j}^{i}\right)^{\prime}(r)\right|_{C_{0}} \ll\left|r^{3}\right|_{C_{0}}, \\
\text { c) } \int_{0}^{1} \frac{k_{j}^{i}(r)}{r^{3}} d r=0, \\
\text { d) } 0<\int_{0}^{c} \frac{k_{j}^{i}(r)}{r^{3}} d r<1 \text { for any } c \text { with } 0<c<1 .
\end{array}\right.
$$

Set $\alpha_{j}^{i}(r)=\frac{1}{r^{3}} \frac{d k_{j}^{i}}{d r}(r)$, which will be smooth on $\mathbb{R}$.

Graphs of typical $\alpha_{j}^{i}$ and $\beta$ are given in Figures 1 and 2 below. 


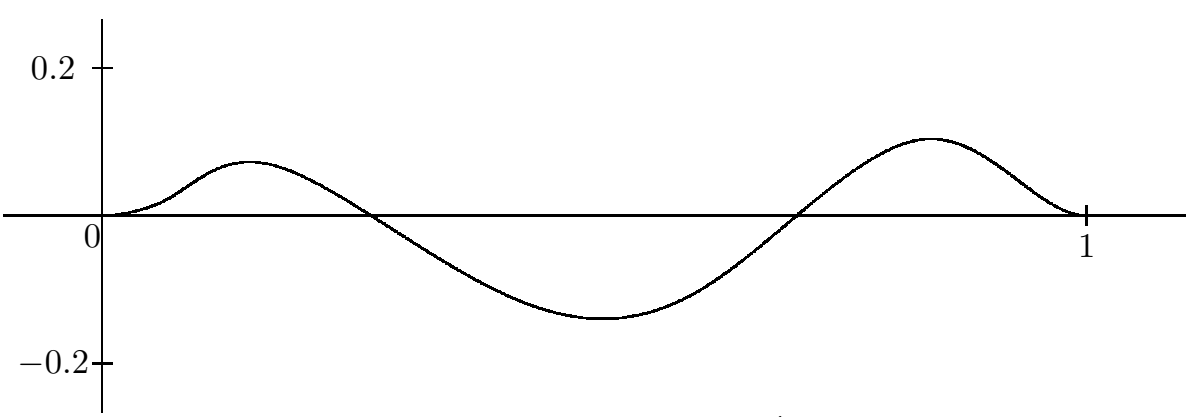

Figure 1. The graph of $\alpha_{j}^{i}$.

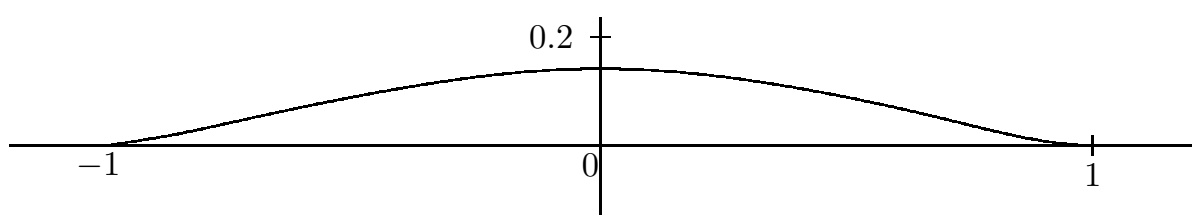

Figure 2. The graph of $\beta$.

Define the functions $F_{i}, i=1, \ldots, n-1$, and $F_{n}$ by

$$
F_{i}\left(r_{1}, \ldots, r_{n}, z\right)=1+\beta(z) \cdot \alpha_{1}^{i}\left(r_{1}\right) \cdots \alpha_{i}^{i}\left(r_{i}\right) \cdots \alpha_{n}^{i}\left(r_{n}\right) \int_{0}^{r_{i}}\left(\frac{1}{y^{3}} \int_{0}^{y} x^{3} \alpha_{i}^{i}(x) d x\right) d y
$$

where ${ }^{\wedge}$ denotes the missing factor in that position,

$$
F_{n}\left(r_{1}, \ldots, r_{n}, z\right)=1-\beta(z) \cdot \sum_{i=1}^{n-1} \alpha_{1}^{i}\left(r_{1}\right) \cdots \alpha_{n-1}^{i}\left(r_{n-1}\right) \int_{0}^{r_{n}}\left(\frac{1}{y^{3}} \int_{0}^{y} x^{3} \alpha_{n}^{i}(x) d x\right) d y .
$$

We consider $F_{i}$ 's and $F_{n}$ defined on $\mathbb{R}^{2 n+1}=\mathbb{R}^{2} \times \cdots \times \mathbb{R}^{2} \times \mathbb{R}$. Then they satisfy the equation (2) and

$$
\begin{array}{ll}
F_{i}, F_{n} \equiv 1 & \text { if } r_{k} \leq 0 \text { or } r_{k} \geq 1 \text { for some } k, \text { or }|z|>1, \\
F_{i}, F_{n}>0 & \text { everywhere. }
\end{array}
$$

We set $\mathbf{C}=\left\{\left(r_{1}, \theta_{1}, \ldots, r_{n}, \theta_{n}, z\right)|| z \mid<1,0 \leq r_{i}<1,0 \leq \theta_{i}<2 \pi\right\}$. We now see that $\tilde{g}$ is Euclidean away from $\mathbf{C}$ and that its scalar curvature $s_{\tilde{g}}$ is negative inside $\mathbf{C}$ except the thin subset $\mathfrak{T}:=\left\{\left(r_{1}, \theta_{1}, \ldots, r_{n}, \theta_{n}, z\right) \in\right.$ $\left.\mathbf{C} \mid F_{i, j}=0, F_{i, 2 n+1}=0,1 \leq i \neq j \leq n\right\}$.

Proposition 2.1. There exist Riemannian metrics on $\mathbb{R}^{2 n+1}, n \geq 2$ such that their scalar curvatures are negative on the pre-compact subset $\mathbf{C} \backslash \mathfrak{T}$ and they are Euclidean away from $\mathbf{C}$.

We need to recall the similar result in even dimensions from Sections 3 and 5 of $[7]$. 
Proposition 2.2. There exist Riemannian metrics on $\mathbb{R}^{2 n}, n \geq 2$ such that their scalar curvatures are negative on a pre-compact subset $\mathbf{K}$ and they are Euclidean away from $\mathbf{K}$.

\section{Decreasing property of the scalar curvature of metrics}

We are going to show that there is a $C^{\infty}$-continuous path $\tilde{g}_{t}$ among the metrics in the previous section such that its scalar curvature $s\left(\tilde{g}_{t}\right)$ is decreasing in $\mathbf{C} \backslash \mathfrak{T}$ and $\tilde{g}_{t}$ is Euclidean in the complement of $\mathbf{C}$.

We set

$$
\left.F_{i}^{t}\left(r_{1}, \ldots, r_{n}, z\right)=1+t \cdot \beta(z) \cdots \alpha_{1}^{i}\left(r_{1}\right) \cdots \alpha_{i}^{i} \hat{(} r_{i}\right) \cdots \alpha_{n}^{i}\left(r_{n}\right) \int_{0}^{r_{i}}\left(\frac{1}{y^{3}} \int_{0}^{y} x^{3} \alpha_{i}^{i}(x) d x\right) d y
$$

where ^ denotes the missing factor in that position,

$$
F_{n}^{t}\left(r_{1}, \ldots, r_{n}, z\right)=1-t \cdot \beta(z) \cdot \sum_{i=1}^{n-1} \alpha_{1}^{i}\left(r_{1}\right) \cdots \alpha_{n-1}^{i}\left(r_{n-1}\right) \int_{0}^{r_{n}}\left(\frac{1}{y^{3}} \int_{0}^{y} x^{3} \alpha_{n}^{i}(x) d x\right) d y
$$

Still under the relation $F_{i}^{t}=\left(f_{i}^{t}\right)^{-2}, i=1, \ldots, n$, we let

$$
\tilde{g}_{t}=d z^{2}+\sum_{i=1}^{n}\left(f_{i}^{t}\right)^{2} d r_{i}^{2}+\frac{r_{i}^{2}}{\left(f_{i}^{t}\right)^{2}} d \theta_{i}^{2}
$$

The scalar curvature is

$$
s_{\tilde{g}_{t}}\left(r_{1}, \ldots, r_{n}\right)=-\frac{1}{4} \sum_{i<j}\left\{\left(\frac{F_{i, j}^{t}}{F_{i}^{t}}\right)^{2} F_{j}^{t}+\left(\frac{F_{j, i}^{t}}{F_{j}^{t}}\right)^{2} F_{i}^{t}\right\}-\frac{1}{4} \sum_{i=1}^{n}\left(\frac{F_{i, 2 n+1}^{t}}{F_{i}^{t}}\right)^{2} .
$$

One can easily check $\left.\frac{d\left(s\left(\tilde{g}_{t}\right)\right)}{d t}\right|_{t=0}=0$ and

$$
\begin{aligned}
\left.\frac{d^{2}\left(s\left(\tilde{g}_{t}\right)\right)}{d t^{2}}\right|_{t=0}= & -\frac{1}{4} \sum_{i<j}\left\{\left.\frac{d^{2}\left(F_{i, j}^{t}\right)^{2}}{d t^{2}}\right|_{t=0}+\left.\frac{d^{2}\left(F_{j, i}^{t}\right)^{2}}{d t^{2}}\right|_{t=0}\right\} \\
& -\left.\frac{1}{4} \sum_{i=1}^{n} \frac{d^{2}\left(F_{i, 2 n+1}^{t}\right)^{2}}{d t^{2}}\right|_{t=0} \\
= & -\frac{1}{2} \sum_{i<j}\left\{\left(F_{i, j}\right)^{2}+\left(F_{j, i}\right)^{2}\right\}-\frac{1}{2} \sum_{i=1}^{n}\left(F_{i, 2 n+1}\right)^{2} \leq 0
\end{aligned}
$$

Note that inside $\mathbf{C}$ the set of points with $\left.\frac{d^{2}}{d t^{2}}\left(s\left(\tilde{g}_{t}\right)\right)\right|_{t=0}=0$ is identical to the set $\mathfrak{T}$. We see that $s\left(\tilde{g}_{t}\right)$ is strictly decreasing only on $\mathbf{C} \backslash \mathfrak{T}$. In order to have the right decreasing property, we need to diffuse the negativity (of scalar curvature) onto a ball containing $\mathbf{C} \backslash \mathfrak{T}$. 


\section{Diffusion of negative scalar curvature onto a ball}

Our argument in this section is similar to that in [8, Section 4], so we avoid some details. We use the following functions; $F_{t, m}(\rho) \in C^{\infty}\left(\mathbb{R}, \mathbb{R}^{\geq 0}\right)$ for $m>0$, $t \geq 0$ defined by $F_{t, m}(\rho)=m \cdot t^{2} \cdot \exp \left(-\frac{100}{\rho}\right)$ on $\mathbb{R}^{>0}$ and $F_{t, m}=0$ on $\mathbb{R}^{\leq 0}$. Also choose an $H \in C^{\infty}(\mathbb{R},[0,1])$ with $H=0$ on $\mathbb{R}^{\geq 1}, H=1$ on $\mathbb{R}^{\leq 0}$ and $H_{\epsilon}^{b}(\rho)=H\left(\frac{1}{\epsilon}(\rho-b)\right)$ for $b>0, \epsilon>0$.

Let $B_{r}(x)$ be the open ball of radius $r$ with respect to $g_{0}$ centered at $x$. We choose a point $p$ and a number $\epsilon_{1}<0.1$ so that $B_{2 \epsilon_{1}}(p) \subset \mathbf{C} \backslash \mathfrak{T}$. Then $s\left(\tilde{g}_{t}\right)<0$ on $B_{\epsilon_{1}}(p)$ when $0<t<c$ for some number $c$.

Let $f_{t, m} \in C^{\infty}\left(\mathbb{R}^{2 n+1}, \mathbb{R}^{\geq 0}\right)$ be $f_{t, m}(q)=F_{t, m}(\rho(q))$, where $\rho$ is the $g_{0^{-}}$ distance from the above point $p$ to $q \in \mathbb{R}^{2 n+1}$ and let $h_{\epsilon}^{b} \in C^{\infty}\left(\mathbb{R}^{2 n+1}, \mathbb{R}^{\geq 0}\right)$ be $h_{\epsilon}^{b}(q)=H_{\epsilon}^{b}(\rho(q))$. We choose $b=9$ and $\epsilon=\epsilon_{1}$. We consider the Riemannian metric $e^{2 \phi_{t}} \tilde{g}_{t}$, where

$$
\phi_{t}(\rho)=f_{t, m}\left(9+\epsilon_{1}-\rho\right) \cdot h_{\epsilon_{1}}^{9}\left(9+\epsilon_{1}-\rho\right)=m t^{2} e^{-\frac{100}{9+\epsilon_{1}-\rho}} h_{\epsilon_{1}}^{9}\left(9+\epsilon_{1}-\rho\right) .
$$

We consider the scalar curvature $s\left(e^{2 \phi_{t}} \tilde{g}_{t}\right)$. We easily get $\left.\frac{d s\left(e^{2 \phi_{t}} \tilde{g}_{t}\right)}{d t}\right|_{t=0}=0$. Using the conformal deformation formula $s\left(e^{2 \phi_{t}} g_{t}\right)=e^{-2 \phi_{t}}\left(s_{g_{t}}+4 n \Delta_{g_{t}} \phi_{t}-\right.$ $\left.2 n(2 n-1)\left|\nabla_{g_{t}} \phi_{t}\right|^{2}\right)$, we calculate as in [8, Section 4] to show that $\left.\frac{d^{2} s\left(e^{2 \phi_{t}} \tilde{g}_{t}\right)}{d t^{2}}\right|_{t=0}$ $<0$ on $B_{9+\epsilon_{1}}(p)$ for small $m>0$. Note that $e^{2 \phi_{t}} \tilde{g}_{t}=g_{0}$ on $\mathbb{R}^{2 n+1} \backslash B_{9+\epsilon_{1}}(p)$.

But due to the boundary $\partial B_{9+\epsilon_{1}}(p)$, we can not yet conclude the existence of a constant $\varepsilon$ such that $s\left(e^{2 \phi_{t}} \tilde{g}_{t}\right)$ is strictly decreasing in the ball $B_{9+\epsilon_{1}}(p)$ for $0 \leq t \leq \varepsilon$.

We continue to follow the argument in [8, Section 4] to show that $\frac{d s\left(e^{2 \phi_{t}} \tilde{g}_{t}\right)}{d t}<$ 0 on $B_{9+\epsilon_{1}}(p) \backslash \overline{B_{9}(p)}$ when $0<t \leq t_{0}$ for some number $t_{0}>0$.

This yields a scalar-curvature melting $g_{t}=e^{2 \phi_{t}} \tilde{g}_{t}$ on $B_{9+\epsilon_{1}}(p)$. By pulling it back by an affine transformation, we can get a scalar-curvature melting on the unit ball.

In even dimensions, we start with the metrics in Proposition 2.2 and proceed similarly as in Section 3 and Section 4 . Then we can get a scalar-curvature melting on the unit ball in $\mathbb{R}^{2 n}, n \geq 2$. This proves Theorem 1.1.

Remark 4.1. The odd dimensional metric in Proposition 2.1 is in fact a contact metric compatible with the standard contact structure on $\mathbb{R}^{2 n+1}$. We suspect our melting can be done in the space of contact metrics. It is very interesting to find a scalar curvature melting of a general metric on a ball, not to mention a Ricci-curvature melting.

\section{Fubini-Study metric}

In this section we demonstrate that the arguments for Euclidean metrics can work similarly for the Fubini-Study metric.

We need to discuss in the context of almost Kähler metrics, which are Riemannian metrics $g$ compatible with a symplectic structure $\omega$, i.e., $\omega(X, Y)=$ 
$g(X, J Y)$ for an almost complex structure $J$, where $X, Y$ are tangent vectors. Here $\omega$ and $g$ determine $J$. One may refer to [2] for some knowledge of almost Kähler geometry needed in this section. In this geometry, for the canonical hermitian connection $\nabla$ determined by $J$ we have the corresponding hermitian scalar curvature $s^{\nabla}$. It proves to be equal to $\frac{1}{2}\left(s^{*}+s\right)$, where $s^{*}$ is the starscalar curvature. It is known that $s^{*}-s=\frac{1}{2}|D J|^{2}$, where $D$ is the Levi-Civita connection. So $s^{\nabla} \geq s$, with equality if and only if $(\omega, g)$ is Kähler .

In $\left[9\right.$, Subsection 4.1], for a toric symplectic manifold $\left(M^{2 n}, \omega\right)$, i.e., a symplectic manifold equipped with an effective Hamiltonian action of an $n$ dimensional torus $T$, M. Lejmi considered $\omega$-compatible $T$-invariant almost Kähler metrics $g$ which have the local expression

$$
g=\sum_{i, j=1}^{n} G_{i j}(z) d z_{i} \otimes d z_{j}+H_{i j}(z) d t_{i} \otimes d t_{j}
$$

where $z_{1}, \ldots, z_{n}$ are moment coordinates corresponding to Hamiltonian vector fields generating $T$ action and $H=\left(H_{i j}\right)$ is a symmetric positive-definite matrix-valued function and $G=\left(G_{i j}\right)$ is the inverse matrix of $H$. In $z, t$ coordinates, $\omega=\sum d z_{i} \wedge d t_{i}$. Any metric of the form (4) is $\omega$-compatible almost Kähler. He computed that $s^{\nabla}=\frac{1}{2}\left(s+s^{*}\right)=-\sum_{i, j=1}^{n} H_{i j, i j}$, where $(\cdot)_{, i j}=\frac{\partial^{2}(\cdot)}{\partial z_{j} \partial z_{i}}$.

Example ([1]). Consider the complex projective space $\mathbb{C P}_{n}$ with the FubiniStudy metric $g_{F S}$ in homogeneous coordinates $\left[z_{0}, z_{1}, \ldots, z_{n}\right]$. We denote the Kähler form by $\omega_{F S}$. The $T^{n}$-action on $\mathbb{C P}_{n}$ given by $\left(y_{1}, \ldots, y_{n}\right) \cdot\left[z_{0}, z_{1}, \ldots, z_{n}\right]$ $=\left[z_{0}, e^{-y_{1} i} z_{1}, \ldots, e^{-y_{n} i} z_{n}\right]$, is Hamiltonian, with moment map $\mu: \mathbb{C P}_{n} \rightarrow \mathbb{R}^{n}$ given by $\mu\left(\left[z_{0}, z_{1}, \ldots, z_{n}\right]\right)=\frac{1}{\|z\|^{2}}\left(\left\|z_{1}\right\|^{2}, \ldots,\left\|z_{n}\right\|^{2}\right)$.

Set $S_{t}:=\left\{\left(x_{1}, \ldots, x_{n}\right) \mid\right.$ each $\left.x_{i}>0, \sum_{i=1}^{n} x_{i}<t\right\} \subset \mathbb{R}^{n}$. Then the image of $\mu$ is the closure of $S_{1} \cdot g_{F S}$ can be expressed as (4) with some $H_{i j}^{0}(z)$.

Proposition 5.1. Given an open set $S_{c}, 0<c<1$, there exists a family of $T^{n}$-invariant almost-Kähler metrics $\left(\omega_{F S}, \bar{g}_{t}\right)$ on $\mathbb{C P}_{n}, 0 \leq t<\epsilon_{2}$ for some number $\epsilon_{2}$, such that

(i) on $\mathbb{C P}_{n}-\mu^{-1}\left(S_{c}\right) ; \bar{g}_{t}=g_{F S}$ for $0 \leq t<\epsilon_{2}$,

(ii) on $\mathbb{C P}_{n} ; \bar{g}_{0}=g_{F S}, s^{\nabla_{\bar{g}_{t}}}=s^{\nabla_{\bar{g}_{0}}}$ and $s\left(\bar{g}_{t}\right) \leq s\left(\bar{g}_{0}\right)$ for $0 \leq t<\epsilon_{2}$,

(iii) $s\left(\bar{g}_{t}\right)<s\left(\bar{g}_{0}\right)$ for $0<t<\epsilon_{2}$ on some open subset $W$ of $\mu^{-1}\left(S_{c}\right)$.

Proof. Set $H_{i j}^{t}(z)=H_{i j}^{0}(z)+t U_{i j}(z)$ and we denote the corresponding metric in (4) by $\bar{g}_{t}$. The condition $s^{\nabla_{\bar{g}_{t}}}=s^{\nabla_{\bar{g}_{0}}}$ is equivalent to $\sum_{i, j=1}^{n}\left\{U_{i j}\right\}_{, i j}=0$. For its solution, choose $U=\left(U_{i j}\right)$ as the diagonal matrix with diagonal entries

$$
\left.U_{i i}(z)=\alpha_{1}^{i}\left(z_{1}\right) \cdots \alpha_{i}^{i} \hat{\left(r_{i}\right.}\right) \cdots \alpha_{n}^{i}\left(z_{n}\right) \int_{0}^{z_{i}}\left(\int_{0}^{y} \alpha_{i}^{i}(x) d x\right) d y \text { for } i=1, \ldots, n-1
$$


where ^ denotes the missing factor in that position,

$$
U_{n n}(z)=-\sum_{i=1}^{n-1} \alpha_{1}^{i}\left(z_{1}\right) \cdots \alpha_{n-1}^{i}\left(z_{n-1}\right) \int_{0}^{z_{n}}\left(\int_{0}^{y} \alpha_{n}^{i}(x) d x\right) d y
$$

where $\alpha_{j}^{i}\left(z_{j}\right), i=1, \ldots, n-1, j=1, \ldots, n$ are smooth functions on $\mathbb{R}$ which satisfy at least $\alpha_{j}^{i}\left(z_{j}\right)=0$ for $z_{j} \leq 0$, or $z_{j} \geq \tilde{c}$ for some $\tilde{c}>0$. This is similar to the solution of the equation (2). Again, one can properly choose $\tilde{c}$ small and $\alpha_{j}^{i}$ so that $U_{i j}$ become smooth functions with compact support in $\mu^{-1}\left(S_{c}\right)$ and that $\bar{g}_{t}, t>0$, is an almost Kähler metric which is non-Kähler, i.e., $\frac{1}{2}|D J|^{2}=s^{*}-s \neq 0$ somewhere. Indeed, either by direct computation on a component of $D J$ or by an argument using [5, Section 4], one can find $\left\{U_{i j}\right\}$ so that near some chosen point $\bar{g}_{t}$ is non-Kähler for any small $t$.

As $\left(\omega_{F S}, g_{F S}\right)$ is Kähler, $s\left(g_{F S}\right)=s^{\nabla_{\bar{g}_{0}}}$. But then, $s^{\nabla_{\bar{g}_{0}}}=s^{\nabla_{\bar{g}_{t}}} \geq s\left(\bar{g}_{t}\right)$ with equality exactly where $\left(\omega, \bar{g}_{t}\right)$ is Kähler. This proves that $s\left(\bar{g}_{t}\right)<s\left(\bar{g}_{0}\right)$ for $0<t<\epsilon_{2}$ on an open pre-compact subset $W$ of $\mu^{-1}\left(S_{c}\right)$.

The metrics $\bar{g}_{t}$ play the same role as those in Propositions 2.1 or 2.2.

Theorem 5.2. Suppose we are given a point $p_{0} \in \mathbb{C P}_{n}$ and a number $r_{0}$ with $0<r_{0}<\frac{1}{2} \operatorname{diameter}\left(g_{F S}\right)$. Then there exists a $C^{\infty}$-continuous path of Riemannian metrics $g_{t}$ on $\mathbb{C P}_{n}$, which exists for $0 \leq t<\varepsilon$ for some number $\varepsilon$ with the following property: $g_{0}=g_{F S}, s\left(g_{\tilde{t}}\right)<s\left(g_{t}\right)$ for $0 \leq t<\tilde{t}<\varepsilon$ in the ball $B_{r_{0} S}^{g_{F}}\left(p_{0}\right)$ of $g_{F S}$-radius $r_{0}$ centered at $p_{0}$ and $g_{t}$ is isometric to $g_{F S}$ in the complement of the ball.

Proof. Since $\left(\mathbb{C P}_{n}, g_{F S}\right)$ is homogeneous, we may choose the coordinates and hamiltonian $T^{n}$ action so that $p_{0}=\mu^{-1}(0, \ldots, 0)$. We choose $c$ so that $\mu^{-1}\left(S_{c}\right) \subset B_{\frac{r_{0}}{2}}^{g_{F S}}\left(p_{0}\right)$ and get $\bar{g}_{t}$ in Proposition 5.1. Choose the smallest natural number $k$ such that $\left.\frac{d^{k} s_{\bar{g}_{t}}}{d t^{k}}\right|_{t=0}$ is not identically zero. This $k$ exists because at each point $s_{\bar{g}_{t}}$ is a rational function of $t$. Then $\left.\frac{d^{j} s_{\bar{g}_{t}}}{d t^{j}}\right|_{t=0} \equiv 0$ for $j=1, \ldots, k-1$. $\left.\frac{d^{k} s_{\bar{g}_{t}}}{d t^{k}}\right|_{t=0} \leq 0$ and $\left.\frac{d^{k} s_{\bar{g}_{t}}}{d t^{k}}\right|_{t=0}(p)<0$ at some $p \in W$. We now apply the argument of Section 4.

We consider a smooth coordinates system $y:=y_{1}, \ldots, y_{2 n}$ on $B_{\frac{3}{2} r_{0}}^{g_{F S}}\left(p_{0}\right)$, which is a topological ball, such that $y(0)=p$ and $B_{r_{0}}^{g_{F S}}\left(p_{0}\right)$ becomes a $y$ coordinates ball of radius, say $R$. Let $g^{0}$ be the Euclidean metric $g^{0}=d y_{1}^{2}+$ $\cdots+d y_{2 n}^{2}$ and $\rho=\sqrt{\sum_{i=1}^{2 n} y_{i}^{2}}$.

From now on, $B_{r}(\cdot)$ means a ball of $g^{0}$-radius $r$ with center at $\cdot$. For some positive number $\epsilon<\frac{R}{10}, B_{2 \epsilon}(p)$ should satisfy $B_{2 \epsilon}(p) \cap\left\{q\left|\frac{d^{k} s_{\bar{g}_{t}}}{d t^{k}}(q)\right|_{t=0}=0\right\}=$ $\emptyset$. Choosing $\epsilon$ further small if necessary, we assume that $B_{R-\epsilon}(p) \supset B_{\frac{r_{0}}{2}}^{g_{F S}}\left(p_{0}\right)$.

Define $F_{t, m}^{d}(x)=m t^{k} e^{-\frac{d}{x}}$. We consider $g_{t}:=e^{2 \phi_{t}} \bar{g}_{t}$, where $\phi_{t}(\rho)=F_{t, m}^{d}(b+$ $\epsilon-\rho) \cdot h_{\epsilon}^{b}(b+\epsilon-\rho)$. We set $b=R-\epsilon . m$ and $d$ shall be determined below. 
The scalar curvature is as follows; $s\left(g_{t}\right)=e^{-2 \phi_{t}} B$, where $B=s_{\bar{g}_{t}}+a_{n} \Delta_{\bar{g}_{t}} \phi_{t}-$ $b_{n}\left|\nabla_{\bar{g}_{t}} \phi_{t}\right|^{2}$ for some positive numbers $a_{n}, b_{n}$ depending on $n$. Then

$$
\frac{d s\left(g_{t}\right)}{d t}=e^{-2 \phi_{t}}\left(-2 \frac{d \phi_{t}}{d t} B+\frac{d s_{\bar{g}_{t}}}{d t}+a_{n} \frac{d \Delta_{\bar{g}_{t}} \phi_{t}}{d t}-b_{n} \frac{d\left|\nabla_{\bar{g}_{t}} \phi_{t}\right|^{2}}{d t}\right) .
$$

We easily get $\left.\frac{d^{j} s\left(g_{t}\right)}{d t^{j}}\right|_{t=0}=0$ for $j=1, \ldots, k-1$ and

$$
\left.\frac{d^{k} s\left(g_{t}\right)}{d t^{k}}\right|_{t=0}=-2 k ! m s_{g_{0}} e^{-\frac{d}{b+\epsilon-\rho}} h_{\epsilon}^{b}(b+\epsilon-\rho)+\left.\frac{d^{k} s_{\bar{g}_{t}}}{d t^{k}}\right|_{t=0}+\left.a_{n} \frac{d^{k} \Delta_{\bar{g}_{t}} \phi_{t}}{d t^{k}}\right|_{t=0} .
$$

On $B_{b+\epsilon}(p)-B_{\epsilon}(p)$, since $h_{\epsilon}^{b}(b+\epsilon-\rho)=1$ we have

$$
\begin{aligned}
\left.\frac{d^{k} s\left(g_{t}\right)}{d t^{k}}\right|_{t=0} & \leq-2 k ! m s_{g_{0}} e^{-\frac{d}{b+\epsilon-\rho}}+\left.a_{n} \frac{d^{k} \Delta_{\bar{g}_{t}} \phi_{t}}{d t^{k}}\right|_{t=0} \\
& =m k !\left(-2 s_{g_{0}} e^{-\frac{d}{b+\epsilon-\rho}}+a_{n} \Delta_{g_{0}} e^{-\frac{d}{b+\epsilon-\rho}}\right) \\
& \leq m k !\left(-2 s_{g_{0}} G-\alpha_{1} G^{\prime \prime}-\alpha_{2} G^{\prime}\right)<0, \text { when } d \text { is large, }
\end{aligned}
$$

where $G(\rho)=e^{-\frac{d}{b+\epsilon-\rho}}$ and $\alpha_{1}, \alpha_{2}$ are some positive numbers and we used Lemmas 5.3 and 5.4 below. On $B_{\epsilon}(p),\left.\frac{d^{k} s_{\bar{g}}}{d t^{k}}\right|_{t=0}<-c_{1}<0$ for some number $c_{1}>0$, so choose $m>0$ small so that $-2 k ! m s_{g_{0}} e^{-\frac{d}{\delta+\epsilon-\rho}} h_{\epsilon}^{b}(b+\epsilon-\rho)+\left.\frac{d^{k} s_{\bar{g} t}}{d t^{k}}\right|_{t=0}+$ $\left.a_{n} \frac{d^{k} \Delta_{\bar{g}_{t}} \phi_{t}}{d t^{k}}\right|_{t=0}<0$.

In sum, we have $\left.\frac{d^{j} s\left(g_{t}\right)}{d t^{j}}\right|_{t=0}=0$ for $j=1, \ldots, k-1$ and $\left.\frac{d^{k} s\left(g_{t}\right)}{d t^{k}}\right|_{t=0}<0$ on $B_{b+\epsilon}(p)$ and $g_{t}=g_{0}$ on $M-B_{b+\epsilon}(p)$. On $\overline{B_{b}(p)}$, there exists $\epsilon_{3}>0$ such that $s\left(g_{t}\right)$ is strictly decreasing for $0 \leq t \leq \epsilon_{3}$. $d$,

On $B_{b+\epsilon}(p)-\overline{B_{b}(p)}, \bar{g}_{t}=g_{0}$. From (5), Lemmas 5.3, 5.4 and 5.5, for large

$$
\begin{aligned}
& e^{2 \phi_{t}} \frac{d s\left(g_{t}\right)}{d t} \\
= & -2 \frac{d \phi_{t}}{d t}\left(s_{g_{0}}+a_{n} \Delta_{g_{0}} \phi_{t}-b_{n}\left|\nabla_{g_{0}} \phi_{t}\right|^{2}\right)+a_{n} \frac{d \Delta_{g_{0}} \phi_{t}}{d t}-b_{n} \frac{d\left|\nabla_{g_{0}} \phi_{t}\right|^{2}}{d t} \\
= & -2 k m t^{k-1} e^{-\frac{d}{b+\epsilon-\rho}}\left(s_{g_{0}}+a_{n} m t^{k} \Delta_{g_{0}} e^{-\frac{d}{b+\epsilon-\rho}}-b_{n} m^{2} t^{2 k}\left|\nabla_{g_{0}} e^{-\frac{d}{b+\epsilon-\rho}}\right|^{2}\right) \\
& +k a_{n} m t^{k-1} \Delta_{g_{0}} e^{-\frac{d}{b+\epsilon-\rho}}-2 k b_{n} m^{2} t^{2 k-1} \mid \nabla_{g_{0}} e^{-\left.\frac{d}{b+\epsilon-\rho}\right|^{2}} \\
\leq & k m t^{k-1}\left\{-2 e^{-\frac{d}{b+\epsilon-\rho}}\left(s_{g_{0}}+a_{n} m t^{k} \Delta_{g_{0}} e^{-\frac{d}{b+\epsilon-\rho}}\right)+a_{n} \Delta_{g_{0}} e^{-\frac{d}{b+\epsilon-\rho}}\right\} \\
\leq & k m t^{k-1}\left(-2 s_{g_{0}} e^{-\frac{d}{b+\epsilon-\rho}}+\frac{a_{n}}{2} \Delta_{g_{0}} e^{-\frac{d}{b+\epsilon-\rho}}\right) \\
\leq & k m t^{k-1}\left(-2 s_{g_{0}} G-\tilde{\alpha_{1}} G^{\prime \prime}-\tilde{\alpha_{2}} G^{\prime}\right)<0 \text { for numbers } \tilde{\alpha_{1}}, \tilde{\alpha_{2}}>0,
\end{aligned}
$$

while $0<t<\epsilon_{4}$ for some $\epsilon_{4}$. This implies that $s\left(g_{t}\right)$ is strictly decreasing for $0 \leq t<\epsilon_{4}$ on $B_{b+\epsilon}(p)-\overline{B_{b}(p)}$. So, $s\left(g_{t}\right)$ is strictly decreasing for $0 \leq t<\varepsilon=$ $\min \left\{\epsilon_{3}, \epsilon_{4}\right\}$ on $B_{b+\epsilon}(p)$. This proves Theorem 5.2. 
For the function $F(t)=e^{-\frac{d}{t}}$ on $\mathbb{R}^{>0}$, one can modify easily Lemma 1.2 in [10] as follows; for $m_{0}, m_{1} \in \mathbb{R}$ and $m_{2}, b \in \mathbb{R}^{>0}$ there exist numbers $d_{0}(b)>0$ and $d_{1}\left(m_{0}, m_{1}, m_{2}, b\right)>0$ such that $F^{(j)}:=\frac{d^{j} F}{d t^{j}}>0$ on $(0, b)$ for $j=0,1,2,3$ if $d \geq d_{0}(b)$ and $m_{2} F^{\prime \prime}+m_{1} F^{\prime}+m_{0} F>0$ on $(0, b)$ if $d \geq d_{1}\left(m_{0}, m_{1}, m_{2}, b\right)$. Since $G^{(j)}(\rho)=(-1)^{j} F^{(j)}(b+\epsilon-\rho)$, we get:

Lemma 5.3. For $m_{0}, m_{1} \in \mathbb{R}$ and $m_{2}, b \in \mathbb{R}^{>0}$, there exists $d_{2}\left(m_{0}, m_{1}, m_{2}, b\right)$ $>0$ such that $m_{2} G^{\prime \prime}+m_{1} G^{\prime}+m_{0} G>0$ on $(\epsilon, b+\epsilon)$ if $d \geq d_{2}\left(m_{0}, m_{1}, m_{2}, b\right)$. And $(-1)^{j} G^{(j)}>0$ on $(\epsilon, b+\epsilon)$ for $j=0,1,2,3$ if $d \geq d_{0}(b)$.

Next, we modify Corollary 2.3 in [10] as follows. Assume that $g$ on a domain $D \subset \mathbb{R}^{n+1}$ fulfill the following two conditions for some $k>1$ : (i) $g_{\text {Eucl }}(\nu, \nu) \leq$ $k^{2} \cdot g(\nu, \nu)$. (ii) The $C^{3}$-norm $\|g\|_{C_{g_{E u c l}^{3}}}(D) \leq k$. Let $H \in C^{\infty}(\mathbb{R}, \mathbb{R})$ be a function with $H^{\prime} \leq 0, H^{\prime \prime} \geq 0$. Then there are constants $a_{1}, a_{2}>0$ depending only on $n$ and $k$ such that $\left(a_{1} H^{\prime \prime}+a_{2} H^{\prime}\right) \circ \pi \leq-\Delta_{g}(H \circ \pi)$ on $D$, where $\pi: \mathbb{R} \times \mathbb{R}^{n} \rightarrow \mathbb{R}$ is the projection. This can be easily verified, following the argument in pp. 660-661 in [10].

We can choose a coordinates system $\left(u_{1}, \ldots, u_{2 n}\right)$ with $u_{1}=\rho$ on a proper subdomain $\tilde{D}$ of $B_{b+\epsilon}(p)-B_{\epsilon}(p)$ so that (i) and (ii) holds with $g_{E u c l}:=d \rho^{2}+$ $d u_{2}^{2}+\cdots+d u_{2 n}^{2}$. Applying the above paragraph to $\left.g_{0}\right|_{\tilde{D}}$ and $G$, we get:

Lemma 5.4. If $d \geq d_{0}(b)$, there are constants $a_{1}, a_{2}>0$ such that $\Delta_{g_{0}} G(\rho) \leq$ $-a_{1} G^{\prime \prime}-a_{2} G^{\prime}$ on $B_{b+\epsilon}(p)-B_{\epsilon}(p)$.

Putting Lemmas 5.3 and 5.4 together;

Lemma 5.5. $\Delta_{g_{0}} G(\rho)<0$ on $B_{b+\epsilon}(p)-B_{\epsilon}(p)$ if $d$ is large.

Remark 5.6. For the Fubini-Study metric, the kernel of $L_{g}^{*}$ on $\mathbb{C P}_{n}$ is trivial. But we do not know if the kernel of $L_{g}^{*}$ is trivial when restricted to a ball. In any case, our construction gives a large amount of deformation, compared to the small deformation of Corvino's, as the latter is based on Implicit Function Theorem.

\section{References}

[1] M. Abreu, Kähler geometry of toric varieties and extremal metrics, Internat. J. Math. 9 (1998), no. 6, 641-651.

[2] V. Apostolov and T. Drăghici, The curvature and the integrability of almost Kähler manifolds: A survey, Symplectic and contact topology: interactions and perspectives (Toronto, ON/Montreal, QC, 2001), 25-53, Fields Inst. Commun., 35, Amer. Math. Soc., Providence, RI, 2003.

[3] R. Beig, P. T. Chruściel, and R. Schoen, KIDs are non-generic, Ann. Henri Poincaré 6 (2005), no. 1, 155-194.

[4] A. L. Besse, Einstein Manifolds, Ergebnisse der Mathematik, 3. Folge, Band 10, Springer-Verlag, 1987.

[5] M. J. Calderbank, L. David, and P. Gauduchon, The Guillemin formula and Kähler metrics on toric symplectic manifolds, J. Symplectic Geometry 1 (2002), no. 4, 767784 . 
[6] J. Corvino, Scalar curvature deformation and a gluing construction for the Einstein constraint equations, Comm. Math. Phys. 214 (2000), no. 1, 137-189.

[7] Y. Kang and J. Kim, Almost Kähler metrics with non-positive scalar curvature which are Euclidean away from a compact set, J. Korean. Math. Soc. 41 (2004), no. 5, 809-820.

[8] Y. Kang, J. Kim, and S. Kwak, Melting of the Euclidean metric to negative scalar curvature in 3 dimension, Bull. Korean Math. Soc. 49 (2012), no. 3, 581-588.

[9] M. Lejmi, Extremal almost-Kähler metrics, Internat. J. Math. 21 (2010), no. 12, 16391662.

[10] J. Lohkamp, Metrics of negative Ricci curvature, Ann. of Math. (2) 140 (1994), no. 3, 655-683.

[11] _ Curvature h-principles, Ann. of Math. (2) 142 (1995), no. 3, 457-498.

Department of Mathematics

SOGANG UNIVERSITY

SEOUl 121-742, Korea

E-mail address: jskim@sogang.ac.kr 